\title{
MRI Vessel Wall Imaging after Intra-Arterial Treatment for Acute Ischemic Stroke
}

\author{
(D) A. Lindenholz, (DI.C. van der Schaaf, (D)A.G. van der Kolk, (D) H.B. van der Worp, (D)A.A. Harteveld, (D) L.J. Kappelle, and \\ (D). Hendrikse \\ i.
}

\section{ABSTRACT}

BACKGROUND AND PURPOSE: Vessel wall imaging is increasingly performed in the diagnostic work-up of patients with ischemic stroke. The aim of this study was to compare vessel wall enhancement after intra-arterial thrombosuction with that in patients not treated with thrombosuction.

MATERIALS AND METHODS: From 2009 to 2017, forty-nine patients with an ischemic stroke underwent 7T MR imaging within 3 months after symptom onset as part of a prospective intracranial vessel wall imaging study. Fourteen of these patients underwent intra-arterial treatment using thrombosuction (intra-arterial treatment group). In the intra-arterial treatment group, vessel walls were evaluated for major vessel wall changes. All patients underwent pre- and postcontrast vessel wall imaging to assess enhancing foci of the vessel wall using coregistered subtraction images. A Wilcoxon signed rank test was performed to test for differences.

RESULTS: In the intra-arterial treatment group, 11 of 14 patients (79\%) showed vessel wall enhancement compared with 17 of 35 patients without intra-arterial treatment (49\%). In the intra-arterial treatment group, more enhancing foci were detected on the ipsilateral side $(n=18.5)$ compared with the contralateral side $(n=3, P=.005)$. Enhancement was more often concentric on the ipsilateral side $(n=8)$ compared with contralateral side $(n=0, P=.01)$. No differences were found in the group without intraarterial treatment between the number and configuration of ipsilateral and contralateral enhancing foci.

CONCLUSIONS: Patients with intra-arterial treatment by means of thrombosuction showed more (concentric) enhancing foci of the vessel wall ipsilateral compared with contralateral to the treated artery than the patients without intra-arterial treatment, suggesting reactive changes of the vessel wall. This finding should be taken into account when assessing vessel wall MR images in patients with stroke.

ABBREVIATIONS: IAT = intra-arterial treatment; MPIR = magnetization prepared inversion recovery

ntra-arterial treatment (IAT) has proved beneficial in selected patients with an anterior circulation acute ischemic stroke. ${ }^{1}$ With IAT, revascularization of the occluded artery by means of mechanical thrombectomy can be achieved in most patients, restoring blood flow to the brain tissue. Although the overall effects of IAT on clinical outcome are well-known, ${ }^{1,2}$ not much is known about the effects of thrombectomy on the local

Received May 12, 2019; accepted after revision January 28, 2020.

From the Departments of Radiology (A.L., I.C.v.d.S., A.G.v.d.K., A.A.H., J.H.) and Neurology and Neurosurgery (H.B.v.d.W., L.J.K.), University Medical Center Utrecht, Utrecht, the Netherlands

The research of Jeroen Hendrikse was funded by the European Research Council under the European Union's Horizon 2020 Program (H2020)/European Research Council Grant agreement No. 637024 (HEARTOFSTROKE).

Please address correspondence to Arjen Lindenholz, MD, MSc, Department of Radiology, University Medical Center Utrecht, Postbox 85500, 3508 GA Utrecht the Netherlands; e-mail: A.Lindenholz@umcutrecht.nl

- Indicates open access to non-subscribers at www.ajnr.org

Indicates article with supplemental on-line table.

http://dx.doi.org/10.3174/ajnr.A6460 intracranial vessel wall. Recently, there has been concern that IAT might damage the arterial vessel wall. ${ }^{3-6}$

Thrombectomy can be performed with different devices: Stent retrievers or thrombosuction devices have been used most often. ${ }^{2}$ Damage to the intracranial vessel wall may be caused by repeated mechanical sheer stress of the stent retriever on the vessel wall ${ }^{4}$ or the negative pressure (up to $-50 \mathrm{~cm} \mathrm{Hg}$ ) when using a thrombosuction device. ${ }^{5,7}$ This damage may consist of dissections, vessel wall edema, and rupture or damage to the endothelium, potentially leading to recurrent thrombosis and distal embolism. ${ }^{3-6}$ Histopathologic preclinical studies have shown endothelial damage to the vessel wall after thrombectomy, more evident with stent-retriever devices than with thrombosuction devices. ${ }^{3-5,8-10}$ In recent MR imaging studies and other imaging studies performed after IAT, damage to a major vessel wall such as dissection or stenosis was only rarely reported. ${ }^{5,8-12}$ However, the arterial vessel wall more often showed contrast enhancement or wall thickening than arteries of patients who did not undergo 
IAT. ${ }^{11-13}$ In these series, patients were treated mostly with stent retrievers, and scanning was performed with $3 \mathrm{~T}$ MR imaging scanners in the acute setting (first day to first week) after IAT. The longer-term effects after IAT performed by thrombosuction have not been reported yet.

Intracranial vessel wall imaging is expected to be increasingly performed in the diagnostic work-up and follow-up of patients with stroke. The aim of this study was to assess the frequency of longer-term vessel wall changes by assessing the presence and severity of intracranial vessel wall enhancement in patients with stroke after IAT with thrombosuction and compare them with patient with stroke without IAT. Potential differences may support the diagnostic interpretation of intracranial vessel wall changes after acute stroke treatment.

\section{MATERIALS AND METHODS Study Population}

From December 2009 to November 2017, patients with an acute ischemic stroke in the anterior circulation who were treated at the neurology department of the University Medical Center Utrecht were screened for inclusion. Patients were retrieved from the ongoing Intracranial Vessel Wall Imaging study (NTR2119; www.trialregister.nl), a prospective vessel wall MR imaging study recruiting patients who presented with clinical symptoms of anterior circulation ischemia (TIA or stroke). ${ }^{14}$ Main inclusion criteria for the current study were age older than 18 years and the possibility of undergoing a 7T MR imaging examination within 3 months after symptom onset. The patients without IAT were selected on the basis of an anterior circulation nonlacunar infarct. Patients with contraindications for MR imaging or for gadolinium-containing contrast agents were excluded, as well as patients with ischemic stroke caused by vasculitis, reversible cerebral vasoconstriction syndrome, small-vessel disease, or secondary to a recent surgical or interventional procedure. Additional exclusion criteria for the current study were primary treatment with a different strategy than a thrombosuction device for the IAT-group to improve study population homogeneity and previous IAT or TIA as final diagnosis for the non-IAT group. Findings of 23 patients without IAT have been published before. ${ }^{15,16}$ These prior articles dealt with sequence development and vessel wall lesion prevalence, whereas in this study, we report longer-term intracranial vessel wall enhancement after IAT using thrombosuction compared with patients not treated with IAT. This study was approved by the institutional review board of our hospital, and all patients gave written informed consent. For all patients, baseline characteristics including age, sex, vascular risk factors, stroke severity expressed using the NIHSS, as well as stroke classification and time intervals between IAT and imaging were collected. For the IAT-group, time intervals between symptom onset and treatment, procedural time, number of passes needed for thrombus removal, and concomitant treatment with IV recombinant tissue-type plasminogen activator (alteplase) were additionally collected.

\section{Treatment}

Treatment was performed as part of standard clinical care. All patients who were eligible for intravenous thrombolysis received IV alteplase within the 4.5-hour time window from symptom onset. IAT was introduced in our center during the study period after the international IAT trial results, and the first patient treated with a thrombosuction device was included in 2014. The main criteria for IAT were derived from the Multicenter Randomized Clinical Trial of Endovascular Treatment for Acute Ischemic Stroke in The Netherlands (MR CLEAN) trial: ${ }^{1}$ 1) a clinical diagnosis of acute ischemic stroke caused by an intracranial anterior circulation occlusion that was visible on CTA, MRA, or DSA; and 2) the ability to perform treatment within 6 hours from symptom onset. All patients in the IAT-group were treated under general anesthesia. A thrombosuction device (Penumbra System $^{\circledR}$, Alameda, California) was used in all patients included in this study. Procedural complications involving the vessel wall, including dissection or perforation, were noted.

\section{Imaging}

Imaging was performed on a 7T whole-body MR imaging system (Philips Healthcare, Best, the Netherlands) with either a 16- or a 32-channel receive coil and a volume transmit/receive coil for transmission (Quad TR; Nova Medical, Wilmington, Massachusetts). Vessel wall visualization at 7T MR imaging has been shown to be superior compared with 3T MR imaging because of a higher contrast-to-noise ratio and image quality. ${ }^{17,18}$ The imaging protocol included a dedicated pre- and postcontrast $3 \mathrm{D}$ whole-brain T1-weighted magnetization-prepared inversion recovery TSE (MPIR-TSE) vessel wall sequence and a TOF-MRA. ${ }^{16}$ For the postcontrast image acquisitions, a gadolinium-containing contrast agent (gadobutrol, Gadovist $1.0 \mathrm{mmol} / \mathrm{mL}$; Bayer Schering Pharma, Berlin, Germany), dose-adjusted to patient weight, was administered intravenously. The TOF-MRA images were used for anatomic verification of the vessels seen on the MPIR-TSE images. The following scan parameters were used for the MPIR-TSE sequence: $\mathrm{FOV}=220 \times 180 \times 13 \mathrm{~mm}^{3}$, which was optimized to $250 \times 250 \times 190 \mathrm{~mm}^{3}$ and satisfactorily tested for equality in vessel wall lesion detection during the study period; ${ }^{16}$ acquired spatial resolution $=0.8 \times 0.8 \times 0.8 \mathrm{~mm}^{3}$; reconstructed spatial resolution $=$ $0.49 \times 0.49 \times 0.49 \mathrm{~mm}^{3} ; \mathrm{TR}=3952 \mathrm{~ms} ; \mathrm{TE}=37 \mathrm{~ms} ; \mathrm{TI}=1375$ ms; flip angle $=120^{\circ}$; readout bandwidth $=935 \mathrm{~Hz}$; and an acquisition time of $10 \mathrm{~min} 40 \mathrm{sec}$. For the small-FOV sequence, the FOV was placed so that the distal intracranial carotid artery and middle cerebral artery were included in the FOV. Scan parameters for the TOF-MRA were as follows: FOV $=190 \times 190 \times 102 \mathrm{~mm}^{3}$, acquired spatial resolution $=0.4 \times 0.5 \times 0.6 \mathrm{~mm}^{3}$, reconstructed spatial resolution $=0.4 \times 0.4 \times 0.3 \mathrm{~mm}^{3}, \mathrm{TR}=21 \mathrm{~ms}, \mathrm{TE}=2.3$ $\mathrm{ms}$, flip angle $=30^{\circ}$, readout bandwidth $=557 \mathrm{~Hz}$, and acquisition time $=9 \min 18 \mathrm{sec}$.

\section{Image Assessment}

Image assessment was performed off-line on a PACS. All images were independently assessed by 2 readers with expertise in reading neurovascular vessel wall images (A.G.v.d.K., 9 years of experience and A.L., 4 years of experience). Readers were blinded to any patient characteristics. The arterial segments that were analyzed included the left and right intracranial ICAs (the clinoid, supraclinoid, and terminal segments) and the left and right MCAs (M1 and M2 segments). Recanalization after IAT was assessed with TICI grading on postprocedural DSA images. ${ }^{19}$ 
First, in the IAT-group postprocedural DSA, 7T MR vessel wall, and 7T MRA images were assessed for dissections and stenoses as major vessel wall changes. Intracranial stenoses were classified into $<50 \%$ stenosis (minor), $50 \%-69 \%$ stenosis (moderate), $70 \%-99 \%$ stenosis (severe), and occlusion. ${ }^{20}$ Second, all 7T MR vessel wall images were assessed for the presence and number of enhancing foci per arterial segment. The mean numbers of enhancing foci of both readers were used for the analyses. Assessment was blinded to IAT and non-IAT. In all patients (IAT-group and non-IAT group), the arteries in the hemisphere ipsilateral to the ischemic infarction were compared with those of the contralateral side. All enhancing foci were further classified as either concentric (circumference of the vessel wall $>50 \%$ enhancing) or eccentric ( $<50 \%$ circumference enhancement) type enhancement. Intracranial atherosclerosis more often shows eccentric vessel wall enhancement, and an inflammatory state of the vessel wall shows most often concentric vessel wall enhancement. ${ }^{21,22}$

The method and cutoff point for the configuration of vessel wall lesion assessment by visual inspection has been described before as a clinically usable tool for vessel wall assessment. ${ }^{21}$ In the assessment of contrast enhancement, a focus was considered enhancing when the signal intensity approximated the signal

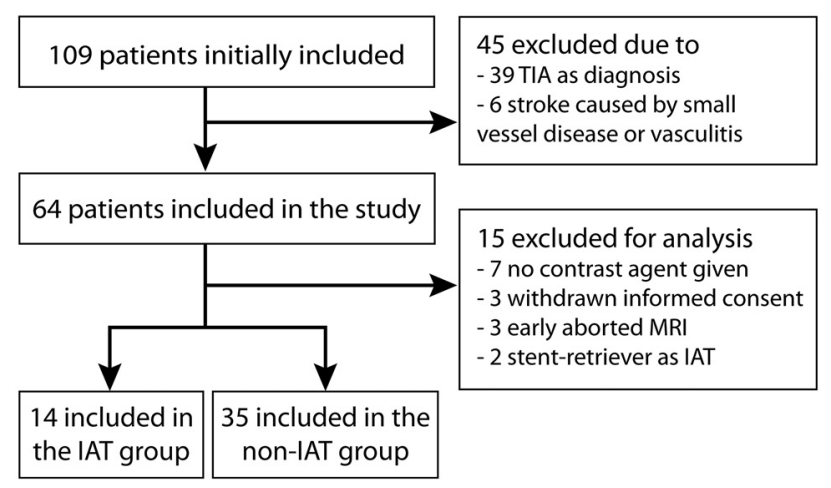

FIG 1. Flowchart of study inclusion. intensity of the (enhancing) pituitary stalk and was present in at least 2 slices. Next, pre- and postcontrast vessel wall images were compared side-by-side to confirm the enhancement. As a double confirmation of enhancement, subtraction images were calculated and used. Thus, pre- and postcontrast vessel wall images were coregistered for the whole $3 \mathrm{D}$ volume using the elastix toolbox in MeVisLab (Version 2.7; MeVis Medical Solutions, Bremen, Germany). ${ }^{23}$ Subsequently, precontrast vessel wall images were subtracted from the coregistered postcontrast vessel wall images and were assessed for contrast enhancement. The registration parameters, $\Delta$ Rotation (in degrees) and $\Delta$ Translation (in millimeters), were used as a measure of motion between pre- and postcontrast vessel wall sequences. The $\Delta$ Rotation and $\Delta$ Translation parameters were calculated as $\sqrt{ }\left(\mathrm{X}\right.$-axis ${ }^{2}+\mathrm{Y}$-axis ${ }^{2}+\mathrm{Z}$-axis $\left.{ }^{2}\right)$. When $>1$ enhancing focus was detected within 1 arterial segment, they were counted separately when they were separated from each other by a normal-appearing vessel wall segment. Also, enhancing foci at the location where the ICA crosses the dura mater from extracranial to intracranial, suspicious for vasa vasorum, were not considered vessel wall enhancement.

\section{Statistical Analysis}

SPSS, Version 21.0 for Windows (IBM, Armonk, New York) was used for statistical analysis. Descriptive statistics were used for the frequency of intracranial dissection or stenosis. Counts are given in proportions (percentages), including their $95 \%$ confidence intervals. The intraclass correlation coefficient using a 2-way mixed, average measurement, consistency model, and the Dice similarity coefficient to correct for the location of the enhancement were calculated to evaluate the interrater agreement. A Wilcoxon signed rank test was used for comparison between the number of enhancing foci ipsilateral and contralateral to the ischemic site as seen on the vessel wall images. A Mann-Whitney $U$ test was performed to compare the number of enhancing foci between the IAT-group and non-IAT group. A 2 -sided $P$ value $<.05$ was considered statistically significant.
Table 1: Baseline characteristics, specified by patients with stroke with and without IAT

\begin{tabular}{|c|c|c|c|}
\hline & IAT Total (\%) & Non-IAT Total (\%) & $P$ Value \\
\hline Age (mean) (range) (yr) & $65(42-85)$ & $60(35-81)$ & $.23^{\mathrm{a}}$ \\
\hline Sex (male) & $10(71 \%)$ & $18(51 \%)$ & $.34^{\mathrm{b}}$ \\
\hline Hypertension & $4(29 \%)$ & $18(51 \%)$ & $.21^{\mathrm{b}}$ \\
\hline Hyperlipidemia & $7(50 \%)$ & $18(51 \%)$ & $.99^{\mathrm{b}}$ \\
\hline Diabetes mellitus & $0(0 \%)$ & 4 (11\%) & $.31^{\mathrm{b}}$ \\
\hline Current smoking & $3(21 \%)$ & $12(34 \%)$ & $.46^{\mathrm{c}}$ \\
\hline Former smoking & $5(36 \%)$ & $7(20 \%)$ & $.46^{\mathrm{c}}$ \\
\hline Atrial fibrillation & $3(21 \%)$ & $4(11 \%)$ & $.39^{\mathrm{b}}$ \\
\hline NIHSS score (mean) (range) & $10.5(3-20)$ & $6.3(0-21)$ & $.02^{\mathrm{a}}$ \\
\hline TOAST criteria $^{30}$ & & & $.62^{c}$ \\
\hline Large-artery atherosclerosis & $7(50 \%)$ & $21(60 \%)$ & \\
\hline Cardioembolism & $4(29 \%)$ & $5(14 \%)$ & \\
\hline Small-vessel occlusion & $0(0 \%)$ & $0(0 \%)$ & \\
\hline Other determined etiology & $1(7 \%)$ & $5(14 \%)$ & \\
\hline Undetermined & $2(14 \%)$ & $4(12 \%)$ & \\
\hline Time to 7T MR imaging (mean) (SD) (day) & $55( \pm 22)$ & $16( \pm 23)$ & $<.001^{\mathrm{a}}$ \\
\hline Time to 7T MR imaging (median) (range) (day) & $58(22-87)$ & $6(1-84)$ & \\
\hline
\end{tabular}

\section{RESULTS}

\section{Patients and Treatment}

Between December 2009 and October 2017, forty-nine patients were included in this study. A flowchart of the study inclusion is shown in Fig 1. Baseline characteristics for both patient groups can be found in the Table 1 .

Fourteen patients underwent IAT using thrombosuction; 2 of them had an occlusion in the distal intracranial ICA, and 12 patients had occlusion in the MCA (Table 2). Twelve patients received intravenous alteplase before intra-arterial mechanical thrombectomy; the other 2 patients exceeded the 4.5hour time window for alteplase treatment. In 2 patients, a stent retriever (Solitaire, Covidien, Irvine, California; or 
Table 2: Location of occlusion and treatment details, including detected enhancing vessel wall foci, in the IAT-group ${ }^{a}$

\begin{tabular}{|c|c|c|c|c|c|c|c|c|c|c|}
\hline $\begin{array}{c}\text { Patient } \\
\text { No. }\end{array}$ & Occlusion Site & $\begin{array}{l}\text { NIHSS } \\
\text { Score }\end{array}$ & NOP & $\begin{array}{c}\text { Time } \\
\text { Symptom } \\
\text { Onset to } \\
\text { IAT (Min) }\end{array}$ & $\begin{array}{l}\text { Procedural } \\
\text { Time (Min) }\end{array}$ & $\begin{array}{l}\text { TICl } \\
\text { Score }\end{array}$ & Alteplase & $\begin{array}{c}\text { Time IAT } \\
\text { to MRI } \\
\text { (Day) }\end{array}$ & $\begin{array}{c}\text { Ipsilateral } \\
\text { Enhancing } \\
\text { Foci }\end{array}$ & $\begin{array}{c}\text { Contralateral } \\
\text { Enhancing } \\
\text { Foci }\end{array}$ \\
\hline 1 & M1 right & 10 & 1 & 190 & 35 & $\mathrm{TICl} 3$ & Yes & 33 & - & - \\
\hline 2 & $\mathrm{M} 1-\mathrm{M} 2$ right & 11 & 1 & 208 & 34 & $\mathrm{TICl} 3$ & Yes & 67 & - & MI-E \\
\hline 3 & Distal carotid left & 8 & 3 & 318 & 50 & $\mathrm{TICl} 2 \mathrm{~b}$ & No & 84 & $\mathrm{Ml}-\mathrm{C}$ & - \\
\hline 4 & M2 left & 5 & 5 & 170 & 83 & $\mathrm{TICl} 2 \mathrm{~b}$ & Yes & 49 & ICA-E, M2-E & ICA-E \\
\hline 5 & M2 left & 5 & 1 & 127 & 42 & $\mathrm{TICl} 3$ & Yes & 65 & - & - \\
\hline 6 & (Large) M3 right & 3 & 1 & 135 & 60 & $\mathrm{TICl} 0$ & Yes & 67 & ICA-C & - \\
\hline 7 & Distal carotid left & 12 & 2 & 201 & 60 & $\mathrm{TICl} 2 \mathrm{~b}$ & Yes & 80 & ICA-C & - \\
\hline 8 & M2 left & 4 & 3 & 275 & 70 & $\mathrm{TICl} 2 \mathrm{~b}$ & No & 59 & ICA-C, MI-E & ICA-E \\
\hline 9 & M1-M2 left & 15 & 1 & 150 & 35 & $\mathrm{TICl} 3$ & Yes & 22 & Ml-E & - \\
\hline 10 & M1 left & 14 & 1 & 150 & 39 & $\mathrm{TICl} 3$ & Yes & 24 & Ml-E & - \\
\hline 11 & M1 right & 12 & 1 & 145 & 29 & $\mathrm{TICl} 3$ & Yes & 25 & $\begin{array}{c}\text { ICA-E 2x, } \\
\text { MI-E }\end{array}$ & - \\
\hline 12 & M1-M2 right & 20 & 1 & 76 & 40 & $\mathrm{TICl} 3$ & Yes & 51 & - & - \\
\hline 13 & M1 right & 15 & 0 & 44 & 42 & $\mathrm{TICl} 3$ & Yes & 57 & - & - \\
\hline 14 & M1-M2 left & 14 & 2 & 180 & 50 & $\mathrm{TICl} 2 \mathrm{~b}$ & Yes & 87 & $\mathrm{Ml}-\mathrm{C}, \mathrm{M} 2-\mathrm{C}$ & - \\
\hline
\end{tabular}

Note:-C indicates concentric; E, eccentric; M, segment of the middle cerebral artery (M1 and M2); NOP, number of passes; -, no enhancing foci detected.

${ }^{a}$ Treatment details of the 14 patients including the number and location of the detected foci of contrast enhancement (by A.G.v.d.K.). In patient 3, the enhancing focus detected in the M1 segment was located distal to the occlusion but directly adjacent to the occlusion site and therefore identified as the same location as the thrombosuction site.
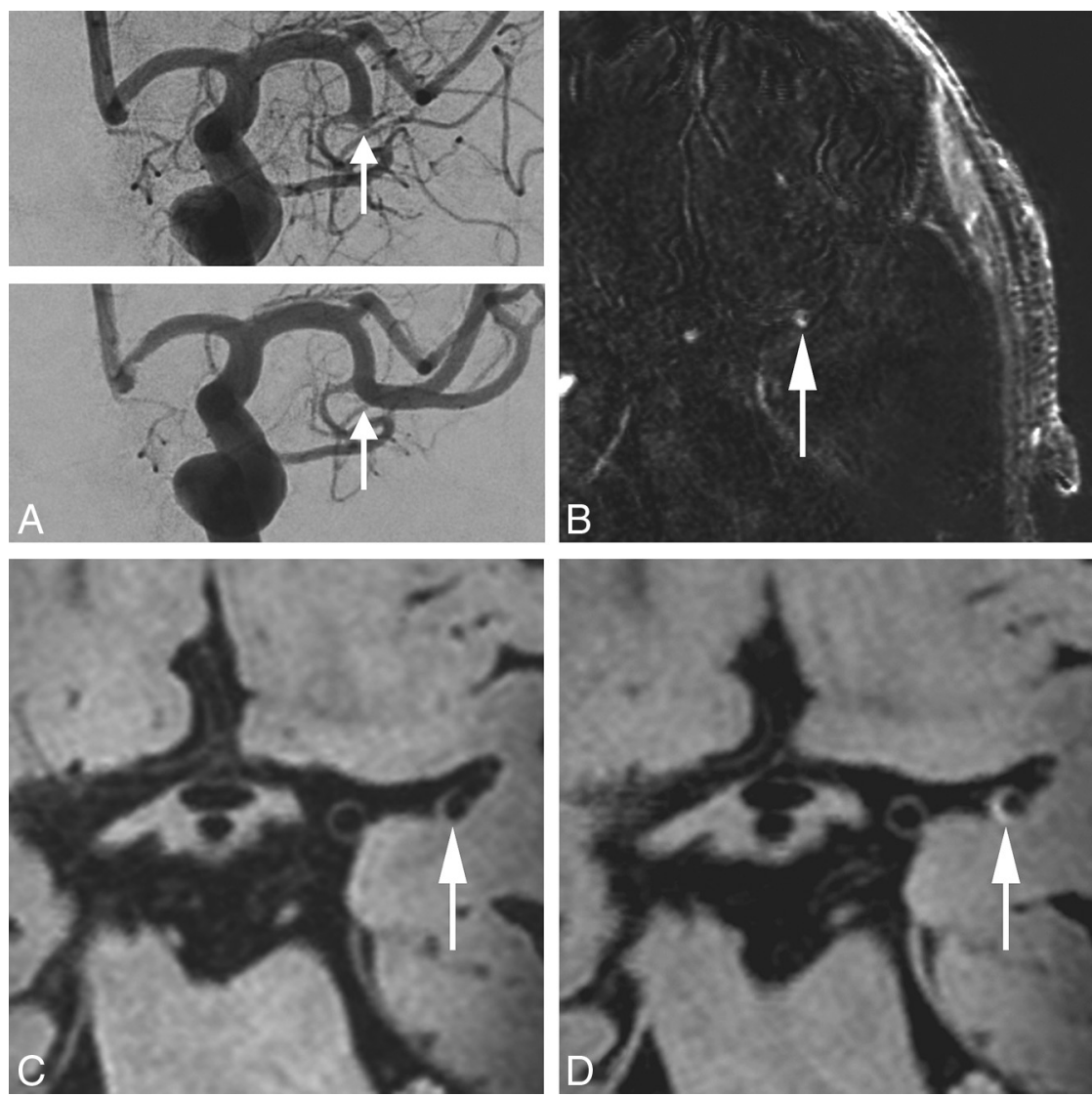

FIG 2. An 85-year-old man with an occlusion of the left M1-M2 segment (arrow), successfully treated with a thrombosuction device (patient 9 in Table 2). A, Digital subtraction angiography directly before and after the thrombectomy procedure. $B$, Subtraction image from coregistered pre- and postcontrast vessel wall images confirms a hyperintense configuration at the thrombectomy site. The transverse pre- $(C)$ and postcontrast $(D)$ MPIR-TSE vessel wall images at 7T (voxel size $=0.8 \times 0.8 \times 0.8 \mathrm{~mm}^{3}$ ) were obtained 22 days after thrombectomy procedure. The eccentric vessel wall enhancement present after contrast administration is at the same location as the thrombectomy site (arrow, D).
Trevo, Stryker, Kalamazoo, Michigan) was used in addition to the thrombosuction device (patients 4 and 14, Table 2). IAT was technically successful in 13 of 14 patients. No procedure-related hemorrhagic complications occurred during the procedures in any of the patients. In 9 patients, 1 pass was sufficient; in the remaining 5 patients, more passes were needed (range, 2-5 passes) to remove the culprit thrombus.

In the non-IAT group, 5 of 35 patients had an ICA infarction (rightsided, $n=3$ ) and 30 patients had an MCA infarction (right-sided, $n=16$ ) (On-line Table). Twelve patients received intravenous alteplase as standard ischemic stroke treatment. In the remaining 23 patients, the 4.5-hour time window was exceeded or only minor symptoms were still present on admission.

\section{Vessel Wall Assessment}

Major Vessel Wall Changes in the IATGroup. Besides a postprocedural moderate stenosis in the treated artery of 1 patient, no other major vessel wall changes were detected. This stenosis in the proximal left M2 segment was detected by MR vessel wall imaging and TOF-MRA ( $>6$ weeks after IAT) at the same location as the removed thrombus and was not yet visible on the postprocedural angiogram obtained in the same session as the 

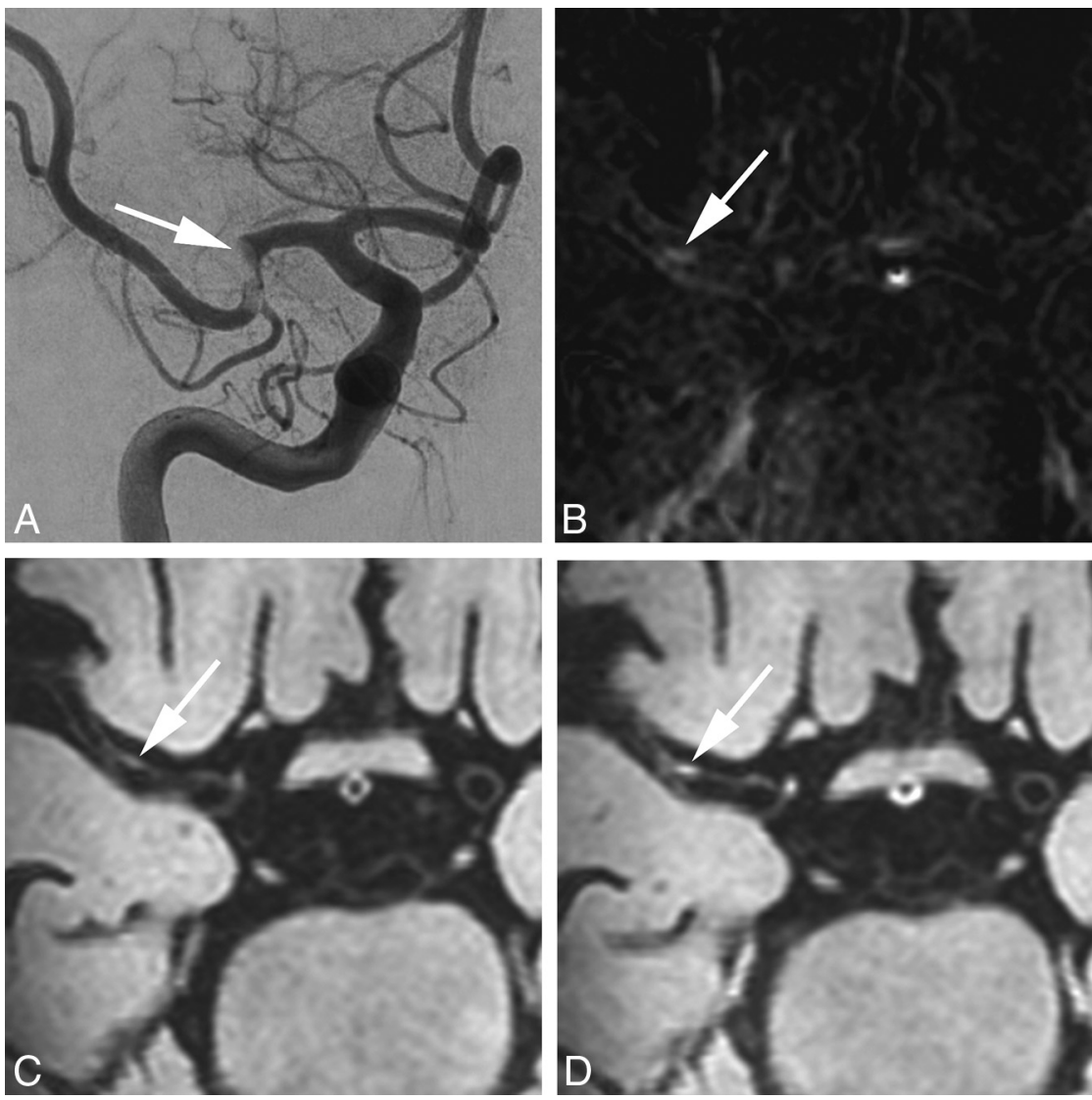

FIG 3. A 72-year-old woman with an occlusion of the right M1 segment, successfully treated with a thrombosuction device (patient 11 in Table 2). Digital subtraction angiography directly before the thrombectomy procedure shows an acute occlusion in the right middle cerebral artery $(A$, arrow). B, Subtraction image from coregistered pre- and postcontrast ( $C$ and $D)$ vessel wall images confirms a hyperintense rim (arrow) at the vessel wall. Transverse pre- $(C)$ and postcontrast (D) MPIR-TSE vessel wall images at 7T were obtained 25 days after the thrombectomy procedure. The area of eccentric vessel wall enhancement $(D)$ is seen in the right M1 segment, at the same location as the thrombectomy site, confirmed on the subtraction image in $B$ (arrows).

enhancement ipsilateral $(n=18.5$, $86 \%)$ to the treated artery compared with the contralateral side $(n=3,14 \%)$ $(P=.005)$. In the non-IAT group, there was no difference in the number of enhancing foci between the ipsilateral $(n=18 ; 60 \%)$ and the contralateral sides $(n=12,40 \%)(P=.47)$. In the IAT-group, vessel wall enhancement was more often concentric on the ipsilateral side $(n=8)$ compared with contralateral side $(n=0)(P=.01)$. In the non-IAT group, there was no significant difference in concentric enhancement between the ipsilateral $(n=7)$ and the contralateral sides $(n=5)(P=.14)$. No differences were found between eccentric enhancing foci on the ipsilateralversus-contralateral side and in the IAT-group versus non-IAT group. The locations of all enhancing foci are shown in Table 2 and the Online Table, and all statistical analyses are shown in Table 3. The degree of movement calculated over all patients was with a mean of $0.77^{\circ} \pm$ $0.51^{\circ}$ for $\Delta$ Rotation and $0.81 \pm 0.59$ $\mathrm{mm}$ for $\Delta$ Translation.

\section{DISCUSSION}

In the current study, we show the patterns of vessel wall changes that can be anticipated in diagnostic vessel wall MR imaging studies in patients with stroke after IAT with a thrombosuc-

thrombectomy. It is not clear whether this was caused by an atherosclerotic plaque or restenosis or was treatment-induced. In patients with a middle cerebral artery occlusion, no T1hyperintensity before contrast was found, nor did we find an MR imaging vessel wall indication suggesting an intramural hemorrhage.

Vessel Wall Enhancement. The interrater agreement for the number and location of the enhancing foci was an intraclass correlation coefficient of 0.91 (95\% CI, 0.84-0.95) and a Dice similarity coefficient of 0.87 . Examples of foci of vessel wall enhancement at the thrombectomy site are shown in Figs 2-4. An example of vessel wall enhancement of the non-IAT group is shown in Fig 5. In the IAT-group, 11 of 14 patients (79\%) showed vessel wall enhancement compared with 17 of 35 patients (49\%) in the nonIAT group. As an average of both readers, in total, 21.5 foci of vessel wall enhancement were detected in the 84 vessel segments (26\%) of the patients with IAT compared with 30 foci of vessel wall enhancement in the 210 vessel segments (14\%) in the patients without IAT (IAT-group versus non-IAT group, $P$ value $=.04)$. In the IAT-group, there were more foci of vessel wall tion device compared with patients with stroke in whom no thrombectomy was performed. MR vessel wall imaging, in the subacute and chronic stages after IAT, showed more foci of vessel wall enhancement on the side treated with thrombectomy, while no difference between ipsi- and contralateral enhancement was found in the patients with stroke without IAT. Additionally, in the IAT-group, these enhancing foci were more often concentric in the vessels ipsilateral to the thrombectomy site compared with the contralateral side.

The higher number of ipsilateral foci of (concentric) contrast enhancement may be explained by several mechanisms: 1) the direct effect of the mechanical forces of the used thrombosuction device; 2) the indirect effect of the removed occluded thrombus by the release of (local) inflammatory molecules; and 3) pre-existent atherosclerotic plaques with possible active inflammation. First, the direct effects of the mechanical forces of the thrombectomy procedure may induce vessel wall changes that cause vessel wall enhancement. This potential explanation is supported by previous imaging that studied the effect of IAT using a stent retriever (and one also including patients treated with a thrombosuction device) on the vessel wall on $3 \mathrm{~T}$ MR imaging. ${ }^{10-13,24}$ The 

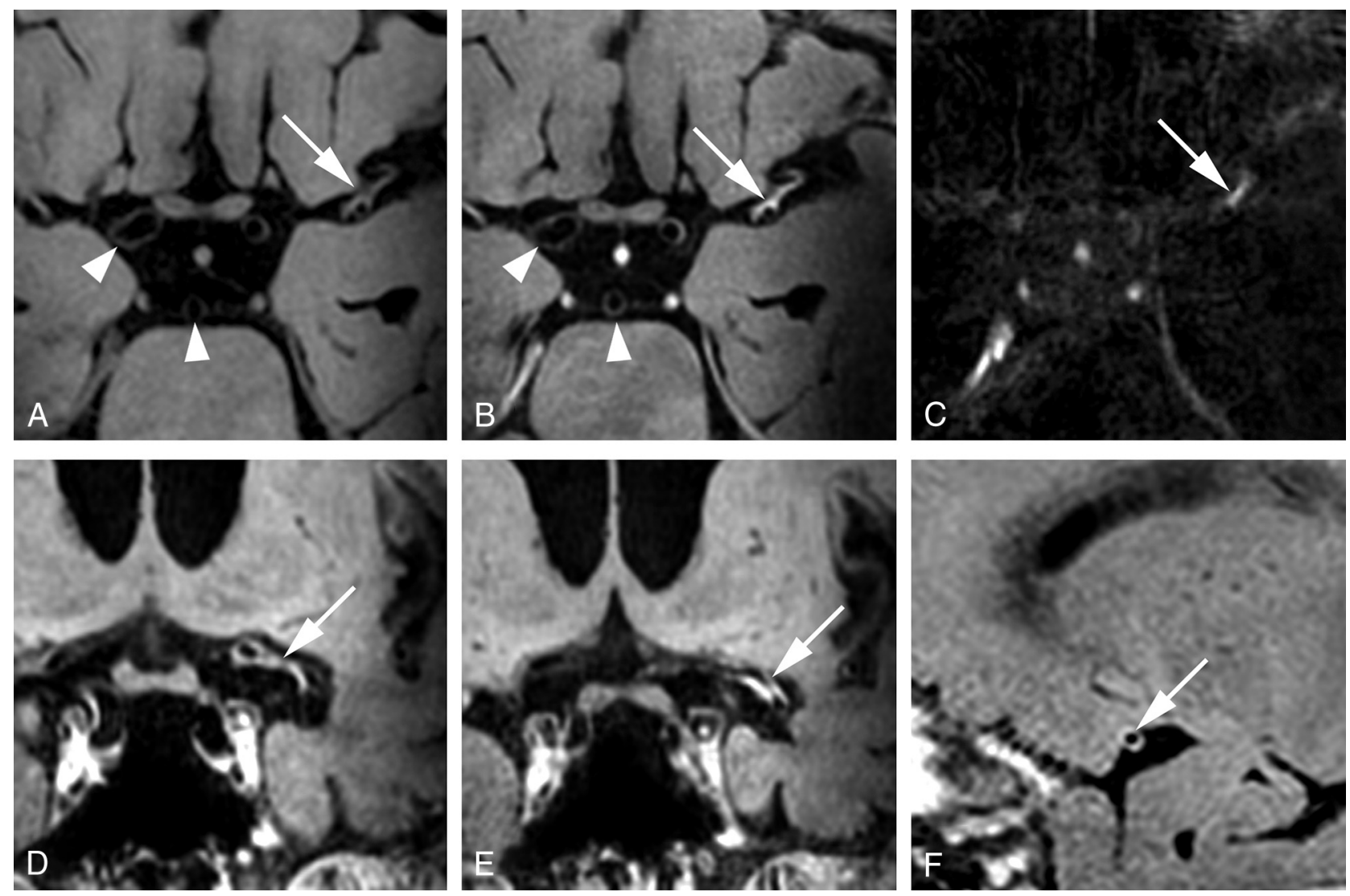

FIG 4. A 67-year-old man with an occlusion of the left M1-M2 segment, successfully treated with intra-arterial thrombectomy (patient 14 in Table 2). The patient was treated with both a stent-retriever device and a thrombosuction device. Axial pre- $(A)$ and postcontrast (B) MPIR-TSE vessel wall images at 7T, 87 days after the thrombectomy procedure. Clear contrast enhancement is present (white arrows) at the same location where the thrombectomy was performed. The carotid and basilar arteries appear normal (white arrowheads, $A$ and $B$ ). $C$, Subtraction image of the pre- and postcontrast vessel wall images confirms the enhancement at the same location. $D$ and $E$, Coronal views of the postcontrast MPIRTSE vessel wall images show enhancement over a long trajectory of the left M1 and M2 segments. F, Sagittal view of the postcontrast MPIR-TSE vessel wall image shows that the enhancement has a concentric configuration.

study that also included patients treated with a thrombosuction device showed that concentric vessel wall enhancement, which occurred in, respectively, $67 \%$ and $14 \%$ of the patients in the IAT-group $(n=15)$ versus the non-IAT group $(n=14)$, was associated with the number of attempts, type of device, and more frequent hemorrhagic transformation of the ischemic infarct (mean interval of treatment to MR imaging, $3.66 \pm 1.52$ days). ${ }^{13}$

In another series (IAT-group, $n=6$, versus non-IAT group, $n=$ $10)$, MR vessel wall imaging showed wall thickening in $83 \%$ versus $30 \%$ and contrast enhancement in $67 \%$ versus $20 \%$ of patients, respectively, (mean interval of treatment to MR imaging, $3.44 \pm$ 2.58 days). ${ }^{11}$ Also, in a recent study, more contrast-enhancing vessel wall areas were found in the post thrombectomy group $(n=6)$, scanned within 24 hours after treatment, compared with a control group $(n=5) .{ }^{12}$ In addition, some follow-up studies (ranging from several days to 2 years) reported delayed arterial vessel wall abnormalities, postulating that these might be attributable to endothelial damage after IAT, $3,6,8,25,26$ On the other hand, in a study with 23 patients with stroke who were scanned within 1 week after thrombectomy, major vessel wall damage such as disruption and stenosis was rare. ${ }^{10}$

We limited our study to patients treated with a thrombosuction device. In this study, 7T MR imaging was used, but previous
3T MR imaging and 7T MR imaging comparison studies showed that $3 \mathrm{~T}$ MR vessel wall imaging can also detect most vessel wall lesions. ${ }^{17,18}$ Furthermore, previous 3T MR imaging studies assessing vessel wall enhancement after treatment with stent-retriever devices have comparable findings. ${ }^{10-13,24}$ Second, the observed vessel wall enhancement may be the result of the former occluding thrombus on the arterial vessel wall that caused reactive, inflammatory changes or a local "scarring" of the vessel wall. This possibility is supported by studies that have shown an increased level of inflammatory markers after an ischemic stroke. ${ }^{27,28}$ A third, more hypothetic explanation for the higher number of ipsilateral foci of contrast enhancement is the presence of pre-existent intracranial atherosclerotic lesions, possibly with active inflammation, in the revascularized segment. ${ }^{29}$ However, the absence of a significant difference in enhancement between the ipsi- and contralateral arteries in our non-IAT group suggests that not all of the observed enhancing foci can be explained by pre-existent atherosclerotic lesions. Furthermore, atherosclerotic lesions often have a more eccentric configuration, which is in contrast to the high number of concentric-type enhancing foci seen in our study and might indicate an inflammatory state of the whole vessel wall rather than an eccentric atherosclerotic plaque. $^{22}$

AJNR Am J Neuroradiol 41:624-31 Apr 2020 www.ajnr.org 
It is unknown how long contrast enhancement of the arterial vessel wall persists after IAT in patients with stroke. In our study, we used a relatively long time interval between thrombectomy and MR imaging (up to 3 months) compared with that in previously published articles (range, 1-11 days). ${ }^{10,11}$ Our results indicate that vessel wall enhancement also persists in the subacute-to-chronic phase.

This study has limitations. First, because local areas of ischemia appear as hypointense parenchymal lesions on the T1weighted vessel wall sequences, we were unable to blind the
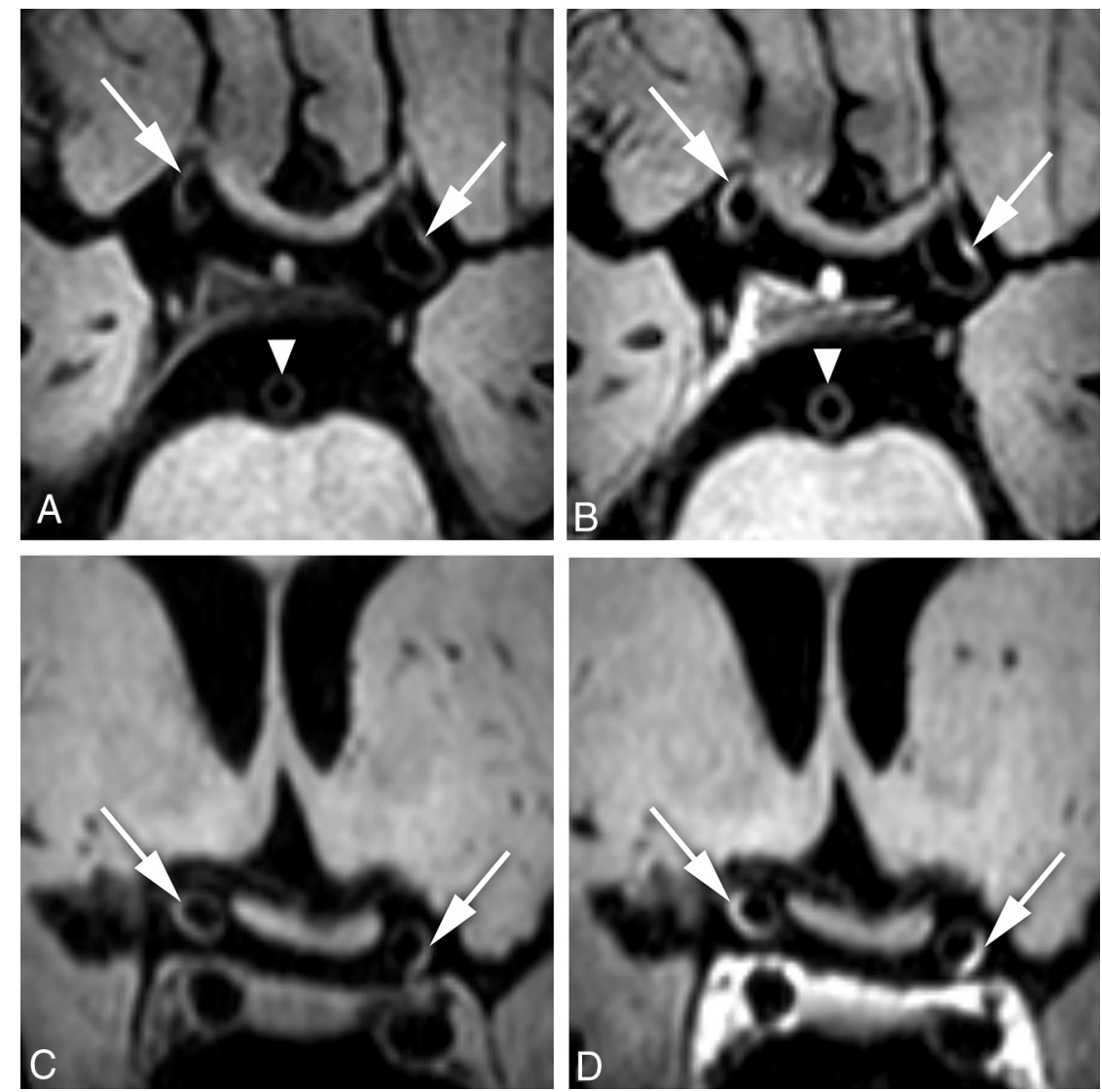

FIG 5. A 75-year-old woman with an anterior circulation ischemic infarction of the left MCA territory (non-IAT group, patient 30 in On-line Table). Transverse and coronal precontrast ( $A$ and $C$ ) and postcontrast $(B$ and $D$ ) MPIR-TSE vessel wall images at 7T were obtained 10 days after symptom onset of the ischemic infarction. Note eccentric vessel wall enhancement of the supraclinoid portion of both intracranial carotid arteries ( $B$ and $D$, arrows). The basilar artery appears normal (white arrowheads, $A$ and $B$ ). observers to the side of thrombectomy in the IAT-group or the side of infarction in the non-IAT group (left or right). Second, IAT was introduced in our center as standard clinical care during the study inclusion period, after the successful international IAT trials. Therefore, the first 28 patients in the non-IAT group did not have IAT as a treatment option. Retrospectively, 10 of all 35 patients without IAT had a proximal occlusion (ICA, M1, or M2) similar to the patients with IAT. In the remaining 25 patients without IAT, no acute occlusion and a better overall NIHSS score was recorded in the final reports (On-line Table). This result may have led to a selection bias in the group without IAT, with the inclusion of lesser affected patients that may have biased the true results. Nevertheless, we believe that the comparison of the ipsilateral-to-contralateral side in the IAT-group is most relevant because in this comparison, all potential individual risk factors are the similar. Third, the number of patients with IAT enrolled in this study is relatively low. Including more patients would benefit the statistical power and enable the possibility of additional analyses. Fourth, for most patients without IAT, the time window between symptom onset and 7T MR imaging was shorter. Nevertheless, there were significantly more enhancing foci in the IAT-group, despite the longer time window. Finally, discrimination between eccentric or concentric enhancing foci can be challenging, and in clinical practice, certainly there is an overlap in the enhancement configuration and the causes of vessel wall enhancement. Histopathologic validation of the detected enhancement would have given insight into the true nature/ composition of the enhancing vessel wall areas (due to the IAT procedure and thrombus-related or pre-existing atherosclerotic plaques); however, this necessitates ex vivo tissue, which was not available. Therefore, some of the

Table 3: Comparison of enhancing foci between the IAT-group and the non-IAT group and between the ipsilateral and contralateral sides

\begin{tabular}{|c|c|c|c|}
\hline & IAT-Group & Non-IAT Group & $P$ Value \\
\hline Total No. of enhancing foci & 21.5 & 30 & $.04^{\mathrm{a}}$ \\
\hline Total No. of ipsilateral enhancing foci & 18.5 & 18.0 & $.003^{\mathrm{a}}$ \\
\hline Total No. of contralateral enhancing foci & 3.0 & 12.0 & $.74^{\mathrm{a}}$ \\
\hline Total No. of concentric ipsilateral foci & 9.0 & 8.5 & $.02^{\mathrm{a}}$ \\
\hline Total No. of eccentric ipsilateral foci & 9.5 & 9.5 & $.07^{\mathrm{a}}$ \\
\hline Total No. of enhancing foci ipsilateral vs contralateral (proportion) $P$ value & $(18.5$ vs 3.0$) .005^{b}$ & $(18.0$ vs 12.0$) .47^{b}$ & \\
\hline Total No. of concentric enhancing foci ipsilateral vs. contralateral (proportion) $P$ value & $(9.0$ vs 0.0$) .011^{\mathrm{b}}$ & (8.5 vs 3.0$) .14^{\mathrm{b}}$ & \\
\hline Total No. of eccentric enhancing foci ipsilateral vs. contralateral (proportion) $P$ value & $(9.5$ vs 3.0$) 06^{\mathrm{b}}$ & $(9.5$ vs 9.0$) .97^{b}$ & \\
\hline
\end{tabular}

\footnotetext{
${ }^{a}$ Mann-Whitney $U$ test.

${ }^{\mathrm{b}}$ Wilcoxon signed rank test.
} 
enhancing foci detected in our study participants might be explained by one of these other mechanisms of wall enhancement. Patients should ideally also have been scanned before the IAT procedure, but due to the limited timeframe in which IAT can be performed, this is difficult to accomplish in clinical practice.

\section{CONCLUSIONS}

IAT using thrombosuction did show more concentric enhancing foci of the vessel wall ipsilateral to the occlusion site compared with the patients without IAT, suggesting reactive changes of the vessel wall. In an era in which MR vessel wall imaging studies are expected to be increasingly performed in the diagnostic work-up and follow-up of patients with acute stroke, the patterns of vessel wall enhancement after thrombectomy need to be known to avoid misinterpretation of these enhancing patterns in the follow-up MR imaging examinations after acute stroke treatment.

Disclosures: Arjen Lindenholz-RELATED: Grant: European Research Council, Comments: The research of Dr Jeroen Hendrikse has received funding from the European Research Council under the European Union's Horizon 2020 Program (H2020)/European Research Council Grant agreement no 637024 (HEARTOFSTROKE).* H. Bart van der Worp_-UNRELATED: Consultancy: Boehringer Ingelheim, Bayer AG, LivaNova, Comments: member of Advisory Board (Boehringer Ingelheim, Bayer AG), outcome adjudication (LivaNova)*; Grants/Grants Pending: European Union, Dutch Heart Foundation, Comments: grants for stroke research.* *Money paid to the institution.

\section{REFERENCES}

1. Berkhemer OA, Fransen PS, Beumer D, et al; MR CLEAN Investigators. A randomized trial of intraarterial treatment for acute ischemic stroke. N Engl J Med 2015;372:11-20 CrossRef Medline

2. Rodrigues FB, Neves JB, Caldeira D, et al. Endovascular treatment versus medical care alone for ischaemic stroke: systematic review and meta-analysis. BMJ 2016;353:i1754 CrossRef Medline

3. Gory B, Bresson D, Kessler I, et al. Histopathologic evaluation of arterial wall response to 5 neurovascular mechanical thrombectomy devices in a swine model. AJNR Am J Neuroradiol 2013;34:2192-98 CrossRef Medline

4. Teng D, Pannell JS, Rennert RC, et al. Endothelial trauma from mechanical thrombectomy in acute stroke: in vitro live-cell platform with animal validation. Stroke 2015;46:1099-1106 CrossRef Medline

5. Peschillo S, Diana F, Berge J, et al. A comparison of acute vascular damage caused by ADAPT versus a stent retriever device after thrombectomy in acute ischemic stroke: a histological and ultrastructural study in an animal model. J Neurointerv Surg 2017;9:743-49 CrossRef Medline

6. Kurre W, Perez MA, Horvath D, et al. Does mechanical thrombectomy in acute embolic stroke have long-term side effects on intracranial vessels? An angiographic follow-up study. Cardiovasc Interv Radiol 2013;36:629-36 CrossRef Medline

7. Penumbra Pivotal Stroke Trial Investigators. The Penumbra pivotal stroke trial: safety and effectiveness of a new generation of mechanical devices for clot removal in intracranial large vessel occlusive disease. Stroke 2009;40:2761-68 CrossRef Medline

8. Renu A, Laredo C, Lopez-Rueda A, et al. Vessel wall enhancement and blood-cerebrospinal fluid barrier disruption after mechanical thrombectomy in acute ischemic stroke. Stroke 2017;48:651-57 CrossRef Medline

9. Gascou G, Lobotesis K, Machi P, et al. Stent retrievers in acute ischemic stroke: complications and failures during the perioperative period. AJNR Am J Neuroradiol 2014;35:734-40 CrossRef Medline

10. Hsieh K, Verma RK, Schroth G, et al. Multimodal 3 Tesla MRI confirms intact arterial wall in acute patients with stroke after stentretriever thrombectomy. Stroke 2014;45:3430-32 CrossRef Medline
11. Power S, Matouk C, Casaubon LK, et al. Vessel wall magnetic resonance imaging in acute ischemic stroke: effects of embolism and mechanical thrombectomy on the arterial wall. Stroke 2014;45:2330-34 CrossRef Medline

12. Abraham P, Scott Pannell J, Santiago-Dieppa DR, et al. Vessel wall signal enhancement on 3-T MRI in acute patients with stroke after stent retriever thrombectomy. Neurosurg Focus 2017;42:E20 CrossRef Medline

13. Seo WK, Oh K, Suh SI, et al. Clinical significance of wall changes after recanalization therapy in acute stroke: high-resolution vessel wall imaging. Stroke 2017;48:1077-80 CrossRef Medline

14. van der Kolk AG, Zwanenburg JJ, Brundel M, et al. Intracranial vessel wall imaging at 7.0-T MRI. Stroke 2011;42:2478-84 CrossRef Medline

15. van der Kolk AG, Zwanenburg JJ, Brundel M, et al. Distribution and natural course of intracranial vessel wall lesions in patients with ischemic stroke or TIA at 7.0 Tesla MRI. Eur Radiol 2015;25:16921700 CrossRef Medline

16. van der Kolk AG, Hendrikse J, Brundel M, et al. Multi-sequence whole-brain intracranial vessel wall imaging at 7.0 Tesla. Eur Radiol 2013;23:2996-3004 CrossRef Medline

17. Harteveld AA, van der Kolk AG, van der Worp HB, et al. High-resolution intracranial vessel wall MRI in an elderly asymptomatic population: comparison of 3T and 7T. Eur Radiol 2017;27:1585-95 CrossRef Medline

18. Zhu C, Haraldsson $\mathrm{H}$, Tian $\mathrm{B}$, et al. High-resolution imaging of the intracranial vessel wall at 3 and $7 \mathrm{~T}$ using $3 \mathrm{D}$ fast spin echo MRI. MAGMA 2016;29:559-70 CrossRef Medline

19. Fugate JE, Klunder AM, Kallmes DF. What is meant by “TICI"? AJNR Am J Neuroradiol 2013;34:1792-97 CrossRef Medline

20. Samuels OB, Joseph GJ, Lynn MJ, et al. A standardized method for measuring intracranial arterial stenosis. AJNR Am J Neuroradiol 2000;21:643-46 Medline

21. Lindenholz A, van der Kolk AG, Zwanenburg JJM, et al. The use and pitfalls of intracranial vessel wall imaging: how we do it. Radiology 2018;286:12-28 CrossRef Medline

22. Swartz RH, Bhuta SS, Farb RI, et al. Intracranial arterial wall imaging using high-resolution 3-Tesla contrast-enhanced MRI. Neurology 2009;72:627-34 CrossRef Medline

23. Klein S, Staring M, Murphy K, et al. Elastix: a toolbox for intensitybased medical image registration. IEEE Trans Med Imaging 2010;29:196-205 CrossRef Medline

24. Li Y, Turan TN, Chaudry I, et al. High-resolution magnetic resonance imaging evidence for intracranial vessel wall inflammation following endovascular thrombectomy. J Stroke Cerebrovasc Dis 2017;26:e96-98 CrossRef Medline

25. Akpinar S, Yilmaz G. Early middle cerebral artery stenosis following stent-assisted thrombectomy. Interv Neuroradiol 2015;21:337-40 CrossRef Medline

26. Eugene F, Gauvrit JY, Ferre JC, et al. One-year MR angiographic and clinical follow-up after intracranial mechanical thrombectomy using a stent retriever device. AJNR Am J Neuroradiol 2015;36:126-32 CrossRef Medline

27. Schuhmann MK, Gunreben I, Kleinschnitz C, et al. Immunohistochemical analysis of cerebral thrombi retrieved by mechanical thrombectomy from patients with acute ischemic stroke. Int J Mol Sci 2016;17:298 CrossRef Medline

28. Jin R, Liu L, Zhang S, et al. Role of inflammation and its mediators in acute ischemic stroke. J Cardiovasc Transl Res 2013;6:834-51 CrossRef Medline

29. Qiao Y, Zeiler SR, Mirbagheri S, et al. Intracranial plaque enhancement in patients with cerebrovascular events on high-spatial-resolution MR images. Radiology 2014;271:534-42 CrossRef Medline

30. Chung JW, Park SH, Kim N, et al. Trial of ORG 10172 in Acute Stroke Treatment (TOAST) classification and vascular territory of ischemic stroke lesions diagnosed by diffusion-weighted imaging. $J$ Am Heart Assoc 2014;3 CrossRef Medline 